\title{
Rectus sheath haematoma: a new set of diagnostic features
}

\author{
D Maharaj, M Ramdass, S Teelucksingh, A Perry, V Naraynsingh
}

Rectus muscle haematoma is a well documented clinical entity, but its diagnosis remains elusive. A haematoma within the rectus sheath produces a painful, tender swelling that can mimic an intraperitoneal mass with features of an acute abdomen. Two patients with rectus haematomas presenting after bouts of prolonged coughing are reported. In both cases, clinical features and ultrasound findings suggested the presence of intraperitoneal pathology. However, in both cases consistent findings in the history and examination pointed towards the diagnosis of a rectus haematoma. It is proposed that these simple clinical criteria are diagnostic of a rectus sheath haematoma, and can thereby avert an unnecessary laparotomy.

$\mathrm{R}$ ectus sheath haematoma is a well recognised but rare condition with a few anecdotal cases reported in the world literature. ${ }^{12}$ It is a well documented complication of blunt abdominal trauma and anticoagulation. ${ }^{3}{ }^{4}$ Other rare associations that have been reported include severe exertion, pregnancy, ${ }^{6}$ insulin injections, ${ }^{7}$ and as a complication of laparoscopic cholecystectomy. ${ }^{8}$ Lee and colleagues reported one case of rectus sheath haematoma due to a paroxysm of coughing during an exacerbation of asthma. ${ }^{9}$

The diagnosis of this condition remains elusive as in the early stages it can mimic an acute abdomen with blood loss and a mass. Hence, a significant number of patients with rectus sheath haematoma have been treated with exploratory laparotomy because of the difficulty in distinguishing between this and other intra-abdominal disorders. ${ }^{10}$ Both ultrasonography and computed tomography have been used to differentiate rectus sheath haematoma from intra-abdominal pathology with a fair degree of success. ${ }^{11}{ }^{12}$ In the Third World setting, however, such diagnostic facilities may not be readily available and doctors must often rely purely on clinical findings. Careful history taking and attention to a few critical points on examination could reliably differentiate a rectus haematoma from intra-abdominal pathology. We believe also, that in combination with Carnett's test, ${ }^{13}$ one can differentiate the two pathologies, and therefore spare the patient an unnecessary laparotomy.

\section{CASE 1}

A 72 year old woman presented with severe abdominal pain after a bout of severe coughing. On examination, she was found to have generalised peritonism and a mass in the right lower abdomen that did not cross the midline, and seemed to be confined by the linea semilunaris. Carnett's sign was found to be positive with the tenderness in the right lower abdomen worse, and additionally, the mass became less pronounced on elevation of her head.

An abdominal ultrasound revealed an $8 \times 5 \times 7 \mathrm{~cm}$ irregular cystic intraperitoneal mass seen superficially in the right iliac fossa, suggestive of an abscess collection (fig 1).

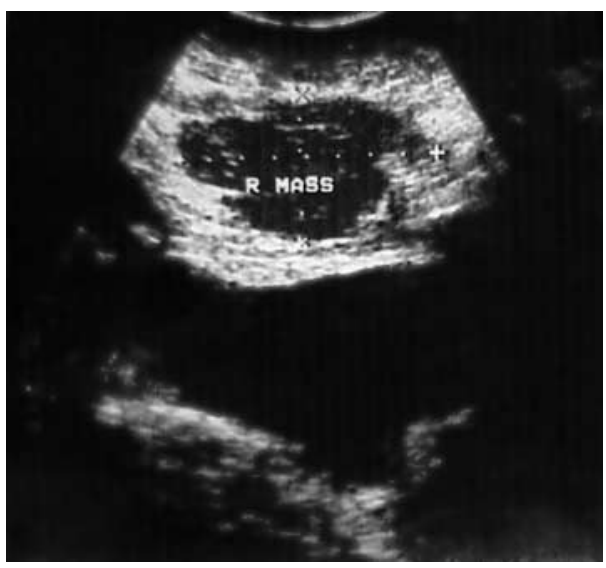

Figure 1 Abdominal ultrasound of case 1.

An exploratory laparotomy was performed which revealed a haematoma of the right rectus muscle, $8 \mathrm{~cm}$ in diameter. No tumour or pus was identified and the inferior epigastric artery was intact. The haematoma was evacuated and the patient recovered uneventfully.

\section{CASE 2}

A 52 year old obese woman presented to the surgical service with features of an acute abdomen and a mass in the right upper quadrant. She had experienced a bout of violent coughing after choking on foodstuff and experienced a sudden tearing sensation in the upper abdomen accompanied by severe pain. Examination revealed a tender mass in the right upper quadrant, the presence of which was exaggerated on elevation of the head, and tenderness exacerbated by contraction of the rectus muscle; these are both features of Carnett's test.

Ultrasound examination of the abdomen confirmed a $5 \times 8$ $\times 3 \mathrm{~cm}$ mass within the rectus sheath. This was evacuated via a small incision over the lesion. Histological evaluation of the rectus muscle biopsy revealed normal muscle tissue with no neoplastic cells. The patient recovered uneventfully.

\section{DISCUSSION}

In 1926, Carnett described a test to differentiate pain originating from the abdominal wall versus pain arising from intra-abdominal pathology. ${ }^{13}$ The test involves placing the patient supine and the site of maximal abdominal tenderness is identified. The patient then sits halfway up and if local palpation at the identified site causes increased tenderness, the test is said to be positive, that is, the cause of the abdominal pain and tenderness is located in the abdominal wall. If the tenderness on palpation of the site in question is alleviated then the pathology is likely to arise from the abdominal viscera as the contracted rectus muscle now protects the intra-abdominal contents. ${ }^{14}$ Causes of abdominal wall tenderness producing a positive Carnett's sign include myositis, 
muscle strain, and nerve entrapment. Thomson and Francis assessed this test in 120 patients and found it to be a reliable means of identifying abdominal wall pathology, saves the expense and dangers of investigation, and even the risk of unnecessary surgery. ${ }^{15}$

The presence of a rectus haematoma also produces a positive Carnett's test as observed in both cases. Contraction of the rectus muscle compresses the haematoma and exacerbates the pain and tenderness.

We wish to make an addition to Carnett's test. With intra-abdominal masses, contraction of the rectus muscle will lead to disappearance or at least a reduced appreciation of it, as the contracted muscle will conceal the mass. For masses arising within the rectus sheath, contraction of the musculature will also make the mass more difficult to palpate, since the anterior rectus sheath becomes taut. We found this sign to be consistent in our cases.

Ultrasonography can be used to differentiate intraperitoneal lesions from extraperitoneal lesions. However, such testing is subject to error, both in obtaining an accurate scan, which may be limited by probe induced tenderness on the acute abdomen, and in interpretation of the images. This is demonstrated by case 1 , in which two qualified ultrasonographers identified the lesion as being "intraperitoneal".

This report also highlights another important point. Violent coughing is a well recognised cause of rib fractures. ${ }^{16}$ The association with rectus haematoma is less recognised. Coughing causes intense contraction of the rectus muscle with tearing and bleeding from the perforating branches of the inferior epigastric vessels within the muscle substance. The occurrence of sudden abdominal pain after severe straining-for example, coughing and sneezing-should alert the clinician to this possibility.

In addition, the condition of rectus haematoma should be suspected in such a patient with the following signs: a mass that is confined by the linea alba and the linea semilunaris, and a positive Carnett's test. Furthermore, the diminution of the mass brought on by contraction of the rectus is diagnostic of any mass arising from or involving the rectus sheath or its contents. These simple features in the history and examination could be used to aid in diagnosis of a rectus haematoma in developing countries where ultrasonography and computed tomography are not readily available. Even in developed coun- tries, where limitations on investigative procedures in not a significant problem, these simple clinical criteria may be used as an adjunct to diagnosis and is particularly attractive as a method of cost containment.

\section{Authors' affiliations}

D Maharai, M Ramdass, S Teelucksingh, A Perry, V Naraynsingh, Department of Surgery, University of the West Indies, General Hospital, Port-of-Spain, Trinidad, West Indies

Correspondence to: Dr Maharaj; dalemaharaj@hotmail.com

Submitted 10 April 2002

Accepted 25 June 2002

\section{REFERENCES}

1 Edlow JA, Juang P, Margulies S, et al. Rectus sheath hematoma. Ann Emerg Med 1999;34:671-5.

2 Berna JD, Garcia-Medina V, Guirao J, et al. Rectus sheath hematoma: diagnostic classification by CT. Abdom Imaging 1996;21:62-4.

3 Berna JD, Zuazu I, Madrigal M, et al. Conservative treatment of large rectus sheath hematoma in patients undergoing anticoagulant therapy. Abdom Imaging 2000:25:230-4.

4 Hegenbarth $\mathbf{R}$, Reiser $C$, Leib P.The sonographic diagnosis of a spontaneous rectus sheath hematoma. Aktuelle Radiol 1991;1:201-3. 5 Hecker RB, Bradshaw WH, Pinkerton SF. Rectus sheath hematoma: report of a case. Tex Med 1990;86:68-70.

6 Humphrey R, Carlan SJ, Greenbaum L. Rectus sheath hematoma in pregnancy. J Clin Ultrasound 2001·29:306-11.

7 Monsein LH, Davis M. Radionuclide imaging of a rectus sheath hematoma caused by insulin injections. Clin Nucl Med 1990;15:539-41.

8 Neufeld D, Jessel J, Freund U. Rectus sheath hematoma: a complication of laparoscopic cholecystectomy. Surg Laparosc Endosc 1992:2:344-5.

9 Lee TM, Greenberger PA, Nahrwold DL, et al. Rectus sheath hematoma complicating an exacerbation of asthma. J Allergy Clin Immunol 1986:78:290-2

10 Miyauchi T, Ishikawa M, Miki H. Rectus sheath hematoma in an elderly woman under anti-coagulant therapy. J Med Invest 2001;48:216-20.

11 Klingler PJ, Wetscher G, Glaser K, et al. use of ultrasound to differentiate rectus sheath hematoma from other acute abdominal disorders. Surg Endosc 1999:13:1129-34.

12 Berna JD, Garcia-Medina V, Guirao J, et al. Rectus sheath hematoma: diagnostic classification by CT. Abdom Imaging 1996;21:62-4.

13 Carnett JB. Intercostal neuralgia as a cause of abdominal pain and tenderness. Surg Gynecol Obstet 1926;42:625-32.

14 Anonymous. Abdominal wall tenderness test: could Carnett cut costs? Lancet 1991;337:1134.

15 Thomson $\mathbf{H}$, Francis DM. Abdominal wall tenderness: a useful sign in the acute abdomen. Lancet 1977;ii: 1053-4.

16 Litch JA, Tuggy M. Cough induced stress fracture and arthropathy of the ribs at extreme altitude. Int J Sports Med 1998;19:220-2. 